\title{
Efficiency of the LDPC Codes in the Reduction of PAPR in Comparison to Turbo Codes and Concatenated Turbo-Reed Solomon Codes in a MIMO-OFDM System
}

\author{
T.Velmurugan ${ }^{\# 1}$, S.Balaji ${ }^{\# 2}$, Anita Sarah Rennie ${ }^{\# 3}$ and D.Sumathi ${ }^{\# 4}$
}

\begin{abstract}
Multiple Input Multiple Output Orthogonal Frequency Division Multiplexing (MIMO-OFDM) systems are the potential candidate for fourth generation (4G) mobile radio communication systems. The MIMO OFDM systems further provide an increase in the data throughput without an increase in the bandwidth and also provide a reduction in the fading. However, the main drawback of OFDM systems is the high peak to average power ratio (PAPR). This disadvantage can be reduced by a number of methods but most of them cause an increase in the bit error rate (BER), data rate loss and an increase in the computational complexity. Thus, the coding methods have been used to reduce the PAPR. LDPC codes were one of the first codes to allow data transmission rates close to the theoretical maximum, the Shannon limit. Although LDPC codes were discovered before turbo codes, the latter was significant in the early years. They have grown to become the standard in the market for highly efficient data transmission methods. These codes show improved performance over an AWGN channel. The Reed Solomon codes, when concatenated with Turbo codes also have been found to improve the performance over wireless channel. However, a comparison of these coding techniques - LDPC, turbo and concatenated turbo-Reed Solomon codes shows that LDPC is most efficient in the reduction of PAPR in a MIMO-OFDM system.
\end{abstract}

Index Terms-PAPR, MIMO, OFDM, LDPC, BER.

\section{INTRODUCTION}

4G or Fourth Generation is the future technology for mobile and wireless communications. The main goal in developing the next generation of wireless communications is increasing the link throughput and network capacity. To achieve this, future systems should be characterized by improved spectral efficiency. Thus, the Multiple Input,

${ }^{1}$ T.Velmurugan,Assistant Professor (Senior), is with the Netwoking Division of School of Electronics Engg. of Vellore Institute of Technology University, Vellore - 14, India (phone: 0416-2202428; cell: 9443215764; e-mail: tvelmurugan@vit.ac.in).

${ }^{2}$ S.Balaji. Assistant Professor (Senior), is with the Applied Electronics Division of School of Electrical Engg. of Vellore Institute of Technology University, Vellore - 14, India (phone: 0416-2202413; cell: 9443436702 ; e-mail: sbalaji@vit.ac.in).

${ }^{3}$ Anita Sarah Rennie is working as Software Engineer in Patni Computer Systems, Mumbai, india and his contact email address is ( Phone : +91-9884686261)

D.Sumathi, Assistant Professor(Jr), is with the Networking Division of School of Information Technology and Engineering of Vellore Institute of Technolgy, Vellore -14, India(phone: 0416-2202428; cell: 9443215764; e-mail: dsumathi@vit.ac.in)
Multiple Output systems came into existence. One of the advantages is that sensitivity to fading is reduced by the spatial diversity provided by multiple spatial paths. OFDM is a digital multi-carrier modulation method where a large number of closely spaced orthogonal subcarriers are used to carry data. . The primary advantage of OFDM over single-carrier schemes is its ability to cope with severe channel conditions - for example, narrowband interference and frequency-selective fading due to multipath. A MIMO-OFDM system transmits independent OFDM modulated data from multiple antennas simultaneously. Thus, a fundamental issue of the OFDM system is also very crucial to the performance of the MIMO-OFDM communication system. One major disadvantage of OFDM is that the time domain OFDM signal which is a sum of several sinusoids leads to high peak to average power ratio (PAPR). Several techniques have been proposed to reduce the high PAPR. In order to reduce the PAPR [1][2] of OFDM systems, many schemes were proposed, among which coding schemes are the most attractive ones due to their inherent error control capability and the simplicity of implementation. The efficiency of the LDPC codes has been compared with turbo codes and concatenated turbo-Reed Solomon codes in a MIMO-OFDM system in this paper.

\section{PAPR OF A SYSTEM}

The peak to average power ratio is an important factor of a communication system. If the peak power is limited by some application or regulatory constraint, the effect is to reduce the average power allowed under multicarrier transmission. This will reduce the range of multicarrier transmission. Moreover, in order to avoid spectral growth of the multicarrier signal in the form of intermodulation among subcarriers, the transmit amplifier would have to be operated in the linear region where there would be a large input backoff and this would cause the power conversion to be inefficient. Thus, a high value of PAPR will force the transmit power amplifier to have a large backoff in order to ensure the linear amplification of the signal while a low value of PAPR will allow the transmit power amplifier to operate efficiently. For the power amplifier to operate efficiently, the peak and average values should be as near to each other as possible.

The PAPR can be calculated by - 


$$
\mathrm{PAPR}=10 \log _{10} \frac{P_{\text {peak }}}{P_{\text {avg }}}
$$

where $\mathrm{P}_{\text {peak }}$ is the maximum power of an OFDM[3] symbol and $\mathrm{P}_{\text {avg }}$ is the average power of an OFDM symbol. When $\mathrm{N}$ subcarriers are added with the same phase in the N-point IFFT stage to produce OFDM symbol, they will almost produce a high peak power equal to $\mathrm{N}$ times the average power. The PAPR can also be written as

$$
\text { PAPR }=10 \log _{10}\left(\frac{\max _{0<t<N T}|x(t)|^{2}}{\frac{1}{N T} \int_{0}^{N T}|x(t)|^{2}}\right)
$$

where $\mathrm{T}$ is the symbol duration, $\mathrm{x}(\mathrm{t})$ is the OFDM symbol at time' $t$ ' which can be expressed as -

$$
\mathrm{x}[\mathrm{t}]=\frac{1}{\sqrt{N}} \sum_{i=0}^{N-1} X[i] e^{j 2 \Pi n i / N}, 0 \leq \mathrm{n} \leq \mathrm{N}-1
$$

and $X[i]$ is the data modulating the $\mathrm{n}^{\text {th }}$ subcarrier.

\section{COMPARISON OF CODING TECHNIQUES USED TO REDUCE THE PAPR PROBLEM}

Low density parity check codes[1] (LDPC) were the first codes to allow data transmission rates close to the theoretical maximum, the Shannon limit. Turbo codes are nowadays competing with LDPC codes which provide similar performance. The main difference between the turbo codes and LDPC codes is that the turbo codes have low encoding complexity and high decoding complexity while the LDPC codes have high encoding complexity and low decoding complexity. The turbo codes and LDPC codes have been shown to perform close to the Shannon limit in the AWGN channel. However, these codes, when concatenated with Reed Solomon codes improve the performance over the wireless channel. Thus, the comparison between the LDPC, Turbo and concatenated Turbo-Reed Solomon codes in reducing the PAPR problem will show which method is most efficient in transmitting data with the least PAPR.

\section{SYSTEM IMPLEMENTATION}

The MIMO - OFDM system begins with the generation of the OFDM signal. The input data stream is firstly encoded and then modulated by a QAM or PSK modulator resulting in a complex symbol stream $\mathrm{X}[0], \mathrm{X}[1], \ldots . . . \mathrm{X}[\mathrm{N}-1]$ which is then passed through a Space Time Block Coding (STBC) MIMO Encoder as part of the MIMO-OFDM modulation block. These symbols are discrete frequency components which are converted into time samples by implementing the Inverse Fast Fourier Transform (IFFT) algorithm. The IFFT produces the OFDM symbol consisting of the sequence $x[n]$ $=x[0], \ldots \ldots ., x[N-1]$ of length $\mathrm{N}$ where -

$$
\mathrm{x}[\mathrm{n}]=\frac{1}{\sqrt{N}} \sum_{i=0}^{N-1} X[i] e^{j 2 \Pi n i / N}, 0 \leq \mathrm{n} \leq \mathrm{N}-1
$$

The multicarrier signal consists of linearly modulated subchannels and the above equation in (4) shows that each
QAM or PSK symbol is modulated by the carrier.The cyclic prefix is then added to the OFDM symbol. The symbols obtained through the serial to parallel converter are passed trough the IFFT stage in the MIMO-OFDM system. Then, the OFDM symbols after reduction of PAPR will be produced through the multiple antennas as the symbols will be spread by the linear coding techniques which spread the symbol in the time domain. The block diagram of the transmitter part of the proposed MIMO-OFDM system is as shown in figure 1.

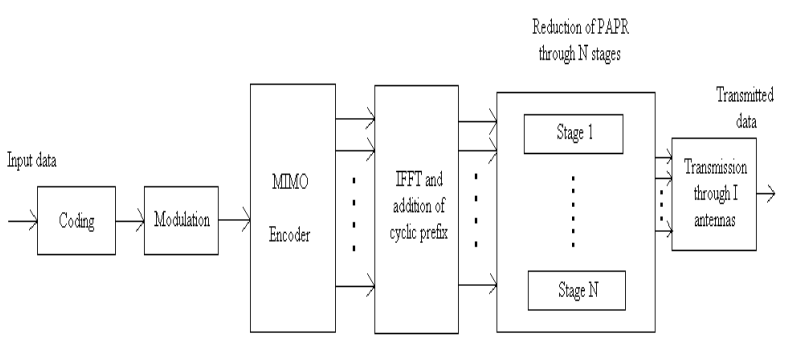

Figure 1: Transmitter of the proposed design

In the MIMO-OFDM[4] system model that has been designed for the reduction of PAPR, turbo coding has been used as the channel coding technique and four modulation techniques - 16 QAM, 64 QAM, BPSK, and QPSK have been used. The Alamouti STBC encoder has been used as the transmitted data is going to be sent through multiple antennas.

The STBC encoder has been used as a MIMO encoder as it helps to transmit multiple copies of a data stream across a number of antennas. The space time block coder helps to achieve transmit diversity. It is the only STBC that can achieve its full diversity gain without needing to sacrifice its data rate and it is especially true for complex modulation symbols.

\section{PAPR REDUCTION METHOD}

The reduction of PAPR occurs through a number of stages. Each symbol is passed through the PAPR reduction method and the process is designed so that replications of the symbol is made by means of the coding technique and the block which has the lowest value of PAPR will be chosen. . The method which has been followed to reduce the PAPR is as follows -

Stage 1: Each of the OFDM symbols is taken and the guard interval is removed. The PAPR is calculated for the OFDM symbol and if it is lower than a particular threshold, it is passed through the STBC encoder from where it will be directly transmitted through the antennas otherwise, it will pass through the reduction technique. The OFDM symbol is then passed through the coding technique after which it would have spread. This happens because the coding techniques used are linear coding techniques and they increase the symbol period in the time domain. The symbol can also be thought to have been spread according to a spreading rate. Thus, it enables us to have a greater chance of each symbol being checked for the lowest PAPR value.

Stage 2: The spread symbol will then be divided into the same number of blocks as the number of antennas. Each block will have the same duration as the original symbol. The 
PAPR of each symbol is calculated and the block with the lowest PAPR will be chosen. The guard interval will be added to the chosen symbol and will be combined with the original OFDM signal. Thus, each symbol is checked and the resulting OFDM signal will consist of all symbols with the lowest PAPR. The final OFDM signal will be transmitted through the antennas.

Instead of using a number of IFFT's according to the number of antennas, the MIMO-OFDM system is implemented in such a way that there is only one IFFT and then the division of each OFDM symbol is done in the PAPR reduction method so that the output produces the same number of blocks as the number of antennas. This reduces the complexity at the transmitter side. The block diagram in figure 2 will easily show how the PAPR reduction occurs for each symbol of the OFDM signal. The transmission occurs such that each symbol of the OFDM signal after being selected as the block which has the lowest PAPR will be transmitted through one of the antennas and the rest of the blocks will be transmitted through the other antennas. This helps in recovering the original data in case the data through any of the antennas get corrupted.

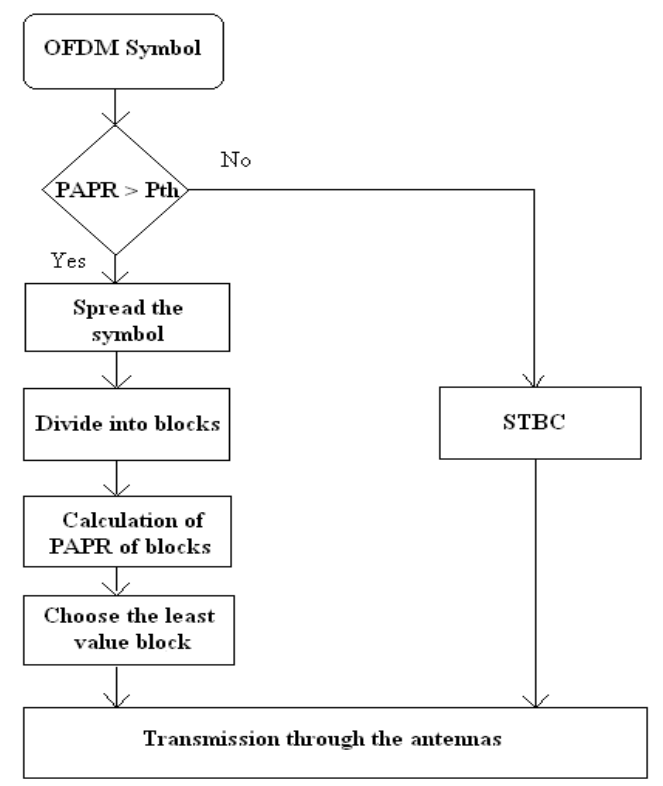

Figure 2: PAPR Reduction Technique

\section{CONSTRUCTION OF LDPC CODING TECHNIQUE}

The LDPC code used in the reduction of PAPR has a parity check matrix which is a block structured matrix which consists of either a zero matrix or a permutation of an identity matrix. The matrix is constructed according to some implementation oriented constraints which leads to better error correcting performance. The entire parity check matrix will be divided into arrays of block matrices each of size $\mathrm{p} \mathrm{x} \mathrm{p}$ and will be denoted as $\mathrm{H}_{\mathrm{i}, \mathrm{j}}$. Each block matrix $\mathrm{H}_{\mathrm{i}, \mathrm{j}}$ is either a zero matrix or a right cyclic shift of an identity matrix. There are certain important parameters that determine the efficiency of the LDPC codes: size of the parity check matrix[5], value of ' $p$ ', node degree distribution and value of ' $g$ '. The parity check matrix for the block LDPC codes to be designed will be as shown in figure 3 . Thus, the code construction parameters for the parity check matrix are - the size of each block matrix is ( $\mathrm{p} x \mathrm{p}$ ), size of each parity check matrix is (m.p $\mathrm{x}$ n.p) and $\mathrm{g}=\gamma . \mathrm{p}$. The row weight distribution is $-\left\{\mathrm{w}_{1}{ }^{(\mathrm{r})}, \mathrm{w}_{2}{ }^{(\mathrm{r})} \ldots \ldots, \mathrm{w}_{\mathrm{m}}{ }^{(\mathrm{r})}\right\}$ where $\mathrm{w}_{\mathrm{i}}{ }^{(\mathrm{r})}$ represents the weight of the $\mathrm{i}^{\text {th }}$ block row. The column weight

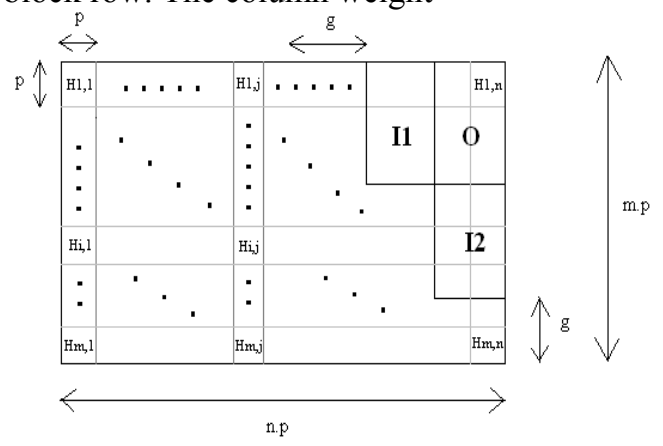

Figure 3: Constructed parity check matrix

distribution is $\left\{\mathrm{w}_{1}{ }^{(\mathrm{c})}, \mathrm{w}_{2}{ }^{(\mathrm{c})} \ldots \mathrm{w}_{\mathrm{n}}{ }^{(\mathrm{c})}\right.$ where $\mathrm{w}_{\mathrm{j}}{ }^{(\mathrm{c})}$ represents the weight of the $j^{\text {th }}$ block column.

The parity check matrix is constructed in the following way - A parity check matrix of the size (m.p x n.p) as shown in figure 3 is created with two identity matrices I1 and I2. There is a zero matrix of the same size as I1. The rest of the matrix is not assigned any value.According to the column weight distribution, a set $\left\{a_{1}, a_{2}, \ldots \ldots \ldots . . a_{n}\right\}$ is generated in which $\mathrm{a}_{\mathrm{j}}=\mathrm{w}_{\mathrm{j}}{ }^{(\mathrm{c})}, 1 \leq \mathrm{j} \leq \mathrm{n}-\mathrm{m}+\gamma$ and $\mathrm{a}_{\mathrm{j}}=\mathrm{w}_{\mathrm{j}}(\mathrm{c})-1, \mathrm{n}-\mathrm{m}+\gamma+1 \leq$ $\mathrm{j} \leq \mathrm{n}$. According to the row weight distribution, a set $\{b 1, b 2, \ldots \ldots \ldots . . b m\}$ is generated in which $-b_{i}=w_{i}^{(r)}-1,1$ $\leq \mathrm{i} \leq \mathrm{m}-\gamma$ and $\mathrm{b}_{\mathrm{i}}=\mathrm{w}_{\mathrm{i}}^{(\mathrm{r})}, \mathrm{m}-\gamma+1 \leq \mathrm{i} \leq \mathrm{m}$. For columns 1 to $\mathrm{n}, \mathrm{a}_{\mathrm{j}}$ null blocks on the $j^{\text {th }}$ block column are replaced with $a_{j}$ right cyclic shifted identity matrices where $\mathrm{i}$ is picked up randomly from the array $i \varepsilon\{1,2,3, \ldots \ldots \ldots . m\}$ such that $b_{i}>0$ and $\mathrm{H}_{\mathrm{i}, \mathrm{j}}$ is a null block. Replace $\mathrm{H}_{\mathrm{i}, \mathrm{j}}$ with a right cyclic shift of a $\mathrm{p} \times \mathrm{p}$ identity matrix. Thus, these steps will lead to the construction of the parity check matrix.

For the encoding procedure[7][8], the parity check matrix can be divided into different sparse matrices as shown below

$$
\left[\begin{array}{lll}
A & B & G \\
C & D & E
\end{array}\right]
$$

where $A$ is $(m-g) x(n-m), B$ is $(m-g) \times g, G$ is $(m-g) x(m-g)$, $\mathrm{C}$ is $\mathrm{g} \times(\mathrm{n}-\mathrm{m}), \mathrm{D}$ is $\mathrm{g} \times \mathrm{g}, \mathrm{E}$ is $\mathrm{g} \times(\mathrm{m}-\mathrm{g})$. All these matrices are sparse and matrix $G$ is lower triangular with ones along the diagonal. Let $\mathrm{x}=\left(\mathrm{s}, \mathrm{p}_{1}, \mathrm{p}_{2}\right)$ be the codeword to be generated where $\mathrm{s}$ is the systematic bit and $\mathrm{p}_{1}, \mathrm{p}_{2}$ combined together form the parity bits. The codeword is made from the matrix in equation where the vector $s$ is of size $(\mathrm{n}-\mathrm{m}), p_{1}$ is of size $(\mathrm{g})$ and $p_{2}$ is of size $(\mathrm{m}-\mathrm{g})$. As $\mathrm{G}$ is lower triangular and all the matrices in the parity check matrix are sparse, the matrix given in equation 5 can be multiplied by an identity matrix as given in [5].Thus, the matrix, when, multiplied from the left by $\left[\begin{array}{cc}I & 0 \\ -E G^{-1} & I\end{array}\right]$ will give the result -

$$
\left[\begin{array}{ccc}
A & B & G \\
-E G^{-1} A+C & -E G^{-1} B+D & 0
\end{array}\right]
$$

It is assumed that the entries of the matrix $\mathrm{H}$ belong to a field F. According to definition, the associated code consists of a set of $\mathrm{n}$ tuples of $\mathrm{x}$ over $\mathrm{F}$ such that $-\mathrm{Hx}^{\mathrm{T}}=0$. Thus, two new equations are obtained - 


$$
\begin{gathered}
\mathrm{As}^{\mathrm{T}}+\mathrm{Bp}_{1}{ }^{\mathrm{T}}+\mathrm{Gp}_{2}{ }^{\mathrm{T}}=0 \\
\left(-\mathrm{EG}^{-1} \mathrm{~A}+\mathrm{C}\right) \mathrm{s}^{\mathrm{T}}+\left(-\mathrm{EG}^{-1} \mathrm{~B}+\mathrm{D}\right) \mathrm{p}_{1}{ }^{\mathrm{T}}=0
\end{gathered}
$$

If the constant before $\mathrm{p}_{1}{ }^{\mathrm{T}}$ in equation (10) is considered to be $\varphi$, then the equation needed to find $\mathrm{p}_{1}{ }^{\mathrm{T}}$ is obtained.

$$
p_{1}{ }^{T}=-\varphi^{-1}\left(-E G^{-1} A+C\right) s^{T}
$$

The determination of $\mathrm{p}_{1}{ }^{\mathrm{T}}$ can be easily computed by breaking the equation into simpler equations. Similarly, the value of $\mathrm{p}_{2}{ }^{\mathrm{T}}$ can be easily computed from equation (9). Thus,

$$
\mathrm{p}_{2}{ }^{\mathrm{T}}=-\mathrm{G}^{-1}\left(\mathrm{As}^{\mathrm{T}}+\mathrm{Bp}_{1}{ }^{\mathrm{T}}\right)
$$

\section{Simulation Results}

The simulation results obtained by the implementation of the coding techniques are analyzed in this chapter. The following parameters have been used for the MATLAB simulation of the reduction of PAPR in the MIMO-OFDM system: a uniformly distributed randomly generated data sequence, channel coding rates of $1 / 2$ and $1 / 3$, modulation techniques - 16 QAM,64 QAM,BPSK and QPSK, 128 point IFFT and STBC MIMO encoder. The various coding techniques that have been used to reduce PAPR are concatenated turbo-Reed Solomon coding[9], turbo coding[5][6][7] and LDPC coding. The Reed Solomon codes have values of $n=15, k=13$. The generator polynomial for

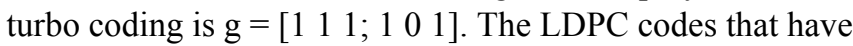
been generated have $\mathrm{m}=64, \mathrm{n}=128, \mathrm{v}=3, \mathrm{~d}_{\text {min }}=6$, two

\begin{tabular}{|c|c|c|c|c|}
\hline $\begin{array}{l}\text { Modulation } \\
\text { technique }\end{array}$ & $\begin{array}{c}\text { No } \\
\text { coding }\end{array}$ & $\begin{array}{l}\text { Turbo- } \\
\text { RS } \\
\text { Coding }\end{array}$ & $\begin{array}{l}\text { Turbo } \\
\text { coding }\end{array}$ & $\begin{array}{l}\text { LDPC } \\
\text { coding }\end{array}$ \\
\hline 16 QAM & 9.86 & 5.25 & 3.59 & 0.8304 \\
\hline 64 QAM & 9.91 & 5.23 & 3.68 & 1.2608 \\
\hline BPSK & 10.52 & 5.28 & 3.76 & 1.1409 \\
\hline QPSK & 10.99 & 5.32 & 3.97 & 1.0209 \\
\hline
\end{tabular}
column weights of 2 and 3, two row weights of 6 and 7. The block matrices have a value of $p=8$. The spreading rate used for these modulation techniques is 3 .

TABLE 2: PAPR REDUCTION (coding rate $=1 / 3$ )

\begin{tabular}{|c|c|c|c|c|}
\hline $\begin{array}{c}\text { Modulation } \\
\text { technique }\end{array}$ & $\begin{array}{c}\text { No } \\
\text { coding }\end{array}$ & $\begin{array}{c}\text { Turbo- } \\
\text { RS } \\
\text { Coding }\end{array}$ & $\begin{array}{c}\text { Turbo } \\
\text { coding }\end{array}$ & $\begin{array}{c}\text { LDPC } \\
\text { coding }\end{array}$ \\
\hline 16 QAM & 10.95 & 5.08 & 3.90 & 1.06 \\
\hline 64 QAM & 10.28 & 5.25 & 3.52 & 1.05 \\
\hline BPSK & 10.84 & 5.29 & 3.90 & 1.06 \\
\hline QPSK & 10.80 & 5.29 & 3.98 & 1.3497 \\
\hline
\end{tabular}

The results given in Table 1 and table 2 shows a comparison between the PAPR obtained from the original OFDM signal and PAPR calculated after using the concatenated turbo-Reed Solomon coding, turbo and LDPC coding techniques to reduce the original value for coding rates $=1 / 2$ and $1 / 3$. The values of PAPR for the no coding scheme in each of the tables range from 9.8-10.9 dB. When the concatenated turbo-Reed Solomon coding method is used, the PAPR values decrease to values of 5.0-5.3 dB for a spreading rate of 3 . The turbo coding techniques are more efficient in that it reduces the PAPR values to 3.5-3.9 dB for a spreading rate of 3 . The LDPC codes are most efficient in the reduction of PAPR in that it gives PAPR values of $0.8-1.3 \mathrm{~dB}$ for a spreading rate of 3 . The graphs plotted are as shown -

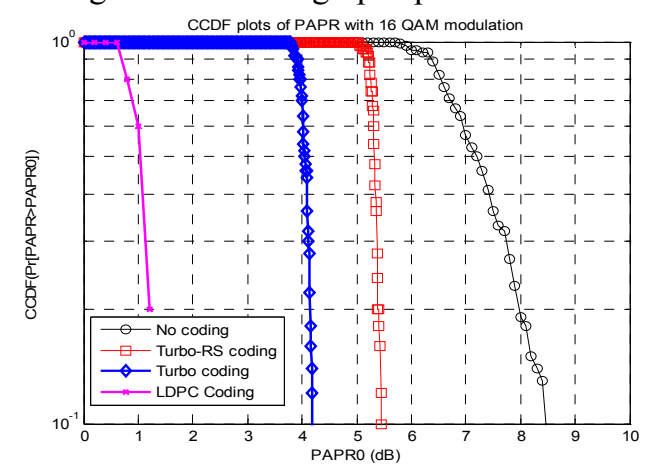

Figure 4: CCDF Plots of PAPR Reduction with 16 Qam Modulation ( coding rate $=1 / 2$, spreading rate $=3$ )

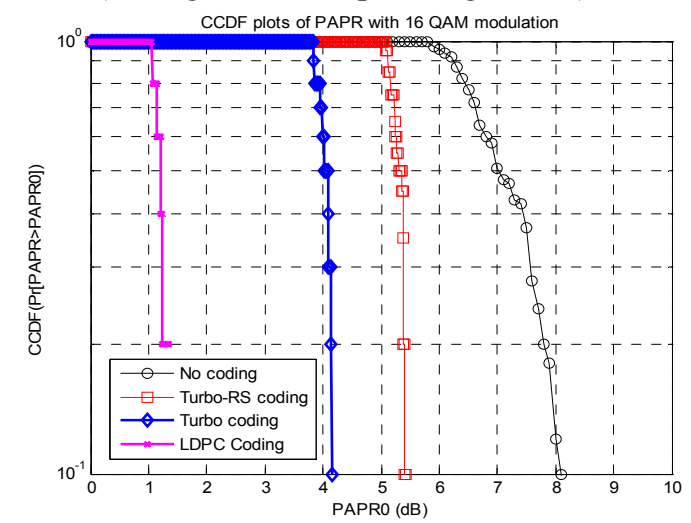

Figure 5: CCDF plots of PAPR reduction with 16 QAM modulation (coding rate $=1 / 3$, spreading rate $=3$ )

In figure 4 , the PAPR value has decreased from the original value to $5.5 \mathrm{~dB}$ in case of concatenated turbo- $\mathrm{RS}$ coding, turbo coding to $4.2 \mathrm{~dB}$ and to $1.2 \mathrm{~dB}$ in case of LDPC coding. In figure 5 , at a CCDF of $10 \%$, it is seen that the PAPR values have reduced to about $5.3 \mathrm{~dB}$ in case of concatenated turbo-RS coding, turbo coding to $4.1 \mathrm{~dB}$ and LDPC coding to about $1.3 \mathrm{~dB}$. It is seen, that the concatenation of the Reed Solomon codes causes an increase in the PAPR value.

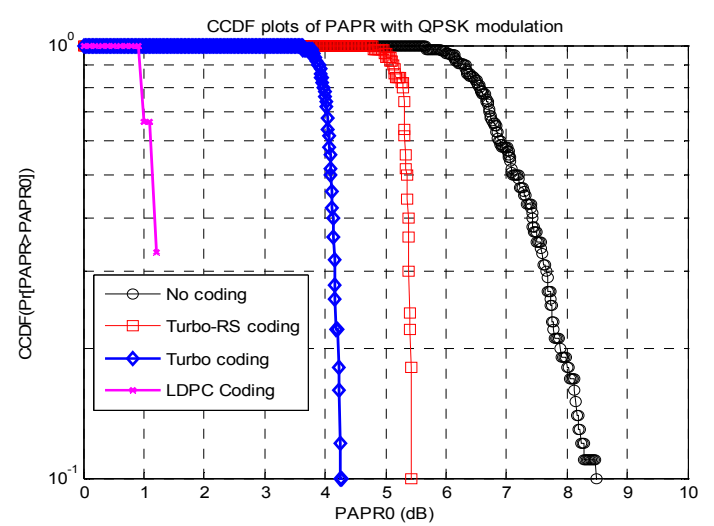

Figure 6: CCDF plots of PAPR reduction with QPSK modulation (coding rate $=1 / 2$, spreading rate $=3$ ) 


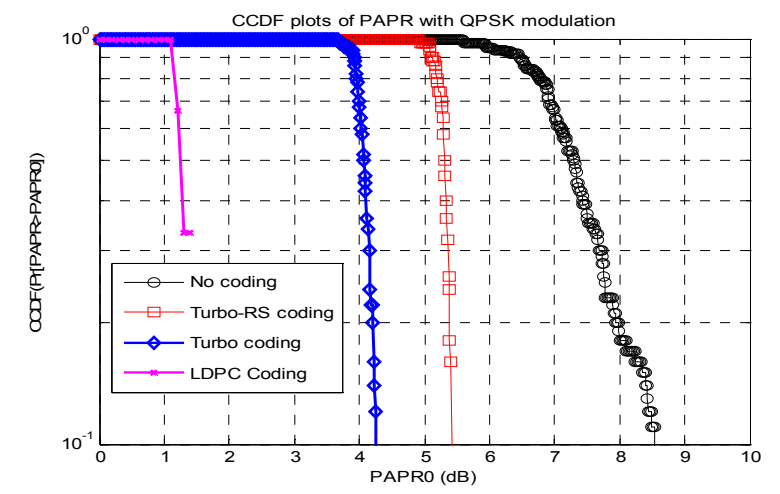

Figure 7: CCDF plots of PAPR reduction with QPSK modulation (coding rate $=1 / 3$, spreading rate $=3$ )

In figure 6 , at a CCDF of $10 \%$, it is seen that the PAPR values have reduced to about $5.5 \mathrm{~dB}$ in case of concatenated turbo-RS coding, turbo coding to $4.2 \mathrm{~dB}$ and LDPC coding to about $1.2 \mathrm{~dB}$. In figure 7 , at a CCDF of $10 \%$, it is seen that the PAPR values have reduced to about $5.5 \mathrm{~dB}$ in case of concatenated turbo-RS coding, turbo coding to $4.2 \mathrm{~dB}$ and LDPC coding to about $1.3 \mathrm{~dB}$.

The concatenated turbo-Reed Solomon coding techniques show a reduction by $47-53 \%$ for a spreading rate of 3 . The turbo coding technique show a reduction of $62-64 \%$ for a spreading rate of 3 . The LDPC coding techniques are the most efficient as the results show that there is a reduction of $87-90 \%$ for a spreading rate of 3 .

\section{CONCLUSION}

This paper has dealt with the reduction of PAPR by use of three coding techniques in the MIMO-OFDM system. As one of the major problems that the OFDM system has to improve upon is the peak to average power ratio, the cause of this problem has been analyzed and a solution has been found out by means of coding techniques. The reduction of this PAPR value can make the system more efficient in addition to the introduction of the MIMO technique.

\section{REFERENCES}

[1] Daoud O., Alani O., "Reducing the PAPR by utilisation of the LDPC code", IET Commun., 2009, Vol. 3, Iss. 4, pp. 520-529.

[2] Al-Akaidi M., Daoud O., "Reducing the peak-to-average power ratio using turbo coding", IEEE Proc. Commun., 2006, 153, (6), pp. 818-821.

[3] Hao Zhong, Tong Zhang, "Block-LDPC: A Practical LDPC Coding System Design Approach", IEEE Transactions on Circuits and Systems-I, Vol.52, No.4, April 2005.

[4] Al-Akaidi M., Daoud O., Gow J., "MIMO-OFDM-based DVBH systems: a hardware design for a PAPR reducing technique", IEEE Trans. Consumer Electron., 2006, 52, (4), pp. 1201-1206.

[5] Richardson T., Urbanke R., "Efficient encoding of low density parity-check codes", IEEE Trans. Information Theory, 2001, 47, (2), pp. $638-656$.

[6] Richardson T., Shokrollahi A., Urbanke R., "Design of capacity-approaching low-density parity-check codes", IEEE Trans. Information Theory, 2001, 47, (2), pp. 619-637.

[7] Zhang T., Parhi K.K., "Joint (3, k)-regular LDPC code and decoder/encoder design”, IEEE Trans. Signal Process., 2004, 52, (4), pp. 1065-1079.

[8] Daoud O., Alani O., "PAPR Reduction by Linear Coding Techniques for MIMO-OFDM Systems Performance Improvement: Simulation and Hardware Implementation", European Journal of Scientific Research, ISSN 1450-216X, Vol.36, No.3 (2009), pp 376-393.

[9] Robert F.H. Fischer, Christian Siegl, "Reed Solomon and simplex codes for peak to average power ratio reduction in OFDM", IEEE Transactions on Information Theory, Vol. 55, No.4, April 2009 